\title{
Transcriptional responses of Bacillus subtillis and thuringiensis to antibiotics and anti-tumour drugs
}

\author{
MOULDY SIOUD, ABDELLATIF BOUDABOUS ${ }^{1}$ and LINA CEKAITE \\ Department of Immunology, The Institute for Cancer Research, \\ The Norwegian Radium Hospital, Montebello, N-0310, Oslo, Norway
}

Received June 11, 2008; Accepted September 5, 2008

DOI: 10.3892/ijmm_00000098

\begin{abstract}
DNA supercoiling is a major regulator of transcription in all organisms. This process is regulated by type I and type II DNA topoisomerases that are targets for microbial antibiotics and/or anti-tumour drugs. Despite extensive studies in this field, no information is available on the response of Bacillus (B) species to eukaryotic DNA topoisomerase inhibitors. Here we found that B.thuringiensis BMG1.7 and HD9 strains are sensitive to DNA gyrase inhibitors (e.g. ciprofloxacin, novobiocin) and to etoposide VP16, a specific inhibitor of eukaryotic type II DNA topoisomerase. Inhibitory or sub-inhibitory concentrations of VP16 induced a drug-tolerant response: an immediate inhibition of growth, followed by a prolonged (10-12 h) lag in growth, and then resumption of normal growth subsequent to overnight culture. Inhibition of the DNA gyrase ATPase activity in $B$. subtillis 168 by novobiocin activated 80 genes and repressed 89 genes at $20 \mathrm{~min}$ after drug addition $(\mathrm{P}<0.05)$. The altered genes belonged to various functional categories dominated by those whose products maintained DNA integrity, mediated transport of low molecular-weight compounds, and SOS response genes. Quantitative RT-PCR revealed comparable effects of novobiocin in B. subtillis and thuringiensis on gene expression. In contrast to novobiocin, VP16 treatment resulted in a moderate effect on $g y r A, g y r B$, and top $A$ gene expression. However, $\operatorname{din} B$ and $\operatorname{lex} A$ genes, involved in SOS response, displayed relatively high transcriptional levels compared to those seen in untreated cells. Furthermore, a small DNA plasmid isolated from novobicin-treated $B$. thuringiensis BMG 1.7 contained a ladder of partially relaxed topoisomers, while the electrophoretic mobility of its counterpart isolated from VP16-treated cells was unchanged. Collectively, the present
\end{abstract}

Correspondence to: Dr M. Sioud, Department of Immunology, The Institute for Cancer Research, The Norwegian Radium Hospital, Montebello, N-0310 Oslo, Norway

E-mail:mosioud@ulrik.uio.no

${ }^{1}$ Present address: Faculty of Sciences, Department of Microbiology, Tunis University, Tunisia

Key words: DNA topoisomerases, anti-tumour drugs, antibiotics, DNA supercoiling, Bacillus subtillis, thuringiensis, microarray, SOS response study would assist in defining common and/or different pathways affected by eubacterial and eukaryotic type DNA topoisomerase inhibitors in the same living organism.

\section{Introduction}

Bacterial chromosomal topology is maintained by the activity of two classes of DNA topoisomerases, type I and II $(1,2)$. These enzymes catalyse transient DNA cleavage and rejoining via a transesterification reaction. Type I enzymes transiently cleave one DNA strand, whereas type II enzymes cleave both strands. Protein $\omega$ and DNA gyrase, type I and II DNA topoisomerases, respectively, are the best characterised activities influencing bacterial DNA supercoiling. Gyrase is composed of two A and two B subunits, encoded by the gyr A and the $\operatorname{gyr} B$ genes, respectively (1). The subunit GyrA catalyses the concomitant double-stranded breakage and rejoining of DNA phosphodiester bonds following binding and hydrolysis of ATP by its partner GyrB. Together with type I DNA topoisomerases, DNA gyrase determines the surpercoiling density of the DNA and solves the topological problems generated during various biological processes including DNA replication, transcription, repair, recombination, and decatenation $(1,2)$.

The biological significance of DNA topoisomerase II is emphasized by the existence of several antibiotics inhibiting their activity. In this respect, DNA gyrase is specifically inhibited by coumarins (e.g. novobiocin) and quinolones, a family of synthetic antimicrobial agents that has been developed for clinical use in patients (e.g. nalidixic acid, ciprofloxacin and norfloxacin)(3). Among the developed fluoroquinolones, ciprofloxacin generally has the greatest activity against gram-negative pathogens (3). As with DNA gyrase, the eukaryotic DNA topoisomerase II is the target of several families of antitumoral drugs, including anthracyclines, acridines, and epipodophyllotoxins (4). All these agents are DNA intercalators with the exception of the epipodophyllotoxins (e.g. etoposide VP16, tenoposide VM26). It should be noted that the quinolones have no activity on eukaryotic type II DNA topoisomerase. Nevertheless, the mechanism of action of quinolones on DNA gyrase and of antitumoral drugs on eukaryotic DNA topoisomerase II have remarkable similarities (5). In the case of DNA gyrase, the interaction of quinolones with GyrA subunit converts DNA gyrase into a lesion-inducing agent, which results in a generation of double- 
stranded DNA breaks. However, the inhibition of the ATPase activity of GyrB by coumarins (e.g. novobiocin) stabilizes the DNA complex without inducing DNA breaks (3).

Previous studies have shown that DNA topoisomerase II inhibitors, which are also DNA intercalators, change the relative binding affinity of this enzyme for different DNA sites, whereas agents that do not intercalate into the DNA, such as quinolones and epipodophyllotoxins, trap DNA topoisomerases II specifically at their physiological sites of action $(3,5,6)$. A direct comparison between the mode of action of quinolones and epipodophyllotoxins has been hampered by the lack of a living organism sensitive to both drugs. We have previously shown that archeabacteria are sensitive to inhibitors of eukaryotic and eubacterial type II DNA topoisomerase $(7,8)$, a finding that correlates well with the intermediate phylogenetic position of archeabacteria between eukaryotes and 'classical bacteria' or eubacteria $(2,9)$. In this respect, we have demonstrated that ciprofloxacin, novobiocin, and etoposide have a common target in Halobacterium GRB: an archaebacterial type II DNA topoisomerase $(10,11)$. Cleavage of pGRB-1 plasmid DNA induced by either ciprofloxacin or the antitumoral drug etoposide VP16, produced DNA fragments of identical lengths, indicating that both drugs trap the reaction intermediates between the archaebacterial DNA topoisomerase and pGRB-1 DNA at the same sites (10). It should be noted that among the archaebacteria, the halobacteria are the most sensitive organisms to DNA topoisomerase II inhibitors (7).

Despite extensive studies in this area, little is known about the sensitivity of B. subtillis and thuringiensis species to eukaryotic DNA topoisomerase inhibitors. In the present study, we report on the effects of novobiocin, ciprofloxacin, and etoposide VP16 on bacterial growth and gene expression.

\section{Materials and methods}

Bacterial strains and drugs. Bacterial strains used in this study were characterized elsewhere $(12,13)$. Strains were cultivated in tryptic soy broth (TSB) medium overnight at $37^{\circ} \mathrm{C}$ with rotary shaking agitation (200 rev/min). Stock cultures were maintained at $-70^{\circ} \mathrm{C}$ with $20 \%$ glycerol. Drugs were purchased from Sigma chemicals. Novobiocin was made up as concentrated stock solutions $(5 \mathrm{mg} / \mathrm{ml})$ in water. Ciprofloxacin was initially dissolved in $0.01 \mathrm{M} \mathrm{NaOH}$ and then diluted in distilled water to $2 \mathrm{mg} / \mathrm{ml}$. Etoposide VP16 was dissolved in dimethylsulfoxide (DMSO) at $50 \mathrm{mg} / \mathrm{ml}$. The used strains were insensitive to DMSO at the concentration used.

Growth and $I C_{50}$ determination. Approximately $40 \mathrm{ml}$ of TSB media were inoculated with $300-500 \mu 1$ of a log-phase culture and several falcon tubes were inoculated with $2 \mathrm{ml}$ and various drug concentrations. After 8 or $24 \mathrm{~h}$ culture at $37^{\circ} \mathrm{C}$ with agitation, growth was determined by measuring the optical density at $600 \mathrm{~nm}$. The $\mathrm{IC}_{50}$ was defined as the concentration of the drug that inhibited growth by $50 \%$ compared with a control culture without the drug. Each drug concentration was tested in triplicate.

Total RNA preparation, cDNA preparation and RT-PCR. Subsequent to drug treatment, bacterial cells were quickly harvested by centrifugation in a microcentrifuge, the supernatants were aspirated and total RNA was isolated using ChargeSwitch ${ }^{\circledR}$ Total RNA isolation kit (Invitrogen) according to the manufacturer's instructions. cDNA synthesis was performed using $2 \mu \mathrm{g}$ of DNase-treated total RNA by random primed reverse transcription in $15 \mu 1$ reaction using First strand cDNA synthesis kit (GE Healthcare). Approximately, $1 \mu 1$ was used for RT-PCR using the following conditions: 25 cycles of denaturation at $94^{\circ} \mathrm{C}$ for $30 \mathrm{sec}$, annealing at $56^{\circ} \mathrm{C}$ for $1 \mathrm{~min}$, and extension at $72^{\circ} \mathrm{C}$ for one min. Primer sequences used were as follows; gyr A, forward primer: 5'CTGTCG TTATGCCTTCAAGGT-3'; reverse primer: 5'GAGTGT CCTGAGTGAGCAGT-3'; gyr A, forward primer 5'-ATAT GCTTGTTGATGGGCATGG-3'; reverse primer: 5'-TCAATGGCGCCTTATTCACTTG-3'; gyr $B$, forward primer: 5'-TGGGGCATCTGTAGTAAATGCT-3'; reverse primer: 5'-CAGGCTCTTCATGGATTGGTTG-3'; topA, forward primer: 5'-GCTTGGCATTTAGCCAATACG-3'; reverse primer: 5'-CTGCCTCTTGTTGCAAGGAA-3'; $\operatorname{din} B$, forward primer: 5'-AGATTGCTATGCACTTGGTTCG-3'; reverse primer: 5'-CCACTTCCCTATCATCCGTACC-3'; lex A, forward primer: 5'-CCAGCAAGACATTCTCGA-3'; reverse primer: 5'-GGTTGTAGACGGAAATGGT-3'; Ribosomal RNA 23S, forward primer: 5'-CCTTAGGAGT CAGACTGCGAGT-3'; reverse primer: 5'-GCACGCTTCT TCTATCCTTCTG-3'. RT-PCR products were separated on $1.5 \%$ agarose gels, visualized by staining with ethidium bromide and photographed using the Molecular Imager ChemiDoc XRS System (BioRad). Band intensities were quantified using Quantity One software.

Analysis of plasmid supercoiling. Treated and untreated cells from $3 \mathrm{ml}$ cultures were harvested $1 \mathrm{~h}$ after novobiocin or VP16 addition. Both drugs were added to the cultures at an $\mathrm{OD}_{600}$ of $\sim 0.4$. Plasmid DNA was prepared using Qiagen's QIAprep Spin Miniprep Kit according to manufacturer's instructions. Plasmid preparations were analyzed by $1 \%$ agarose gels in TAE buffer at $80 \mathrm{~V}$ for $3 \mathrm{~h}$. The gels were stained with ethidium bromide $(0.5 \mu \mathrm{g} / \mathrm{ml})$ and photographed using the Molecular Imager ChemiDoc XRS System (BioRad).

Profiling of gene expression. For all experiments, overnight culture was diluted $1 / 1000$ in $50-100 \mathrm{ml}$ medium and then cells were cultured at $37^{\circ} \mathrm{C}$ with agitation. Drugs were added to the culture at an $\mathrm{OD}_{600}$ of $\sim 0.4$. Untreated cells were cultured in TBS medium with no drug addition. Samples for microarray analysis were taken at 5 and 20 min post drug addition. Each treatment was performed in triplicate. Cell pellets from each triplicate were collected and total RNA was prepared with ChargeSwitch ${ }^{\circledR}$ Total RNA isolation kit (Invitrogen) according to manufacturer's instructions. This method includes one step treatment with DNase I in order to remove contaminating DNA. Fluorescence-labeled cDNAs were synthesized from $10 \mu \mathrm{g}$ of total RNA using SuperScript ${ }^{\mathrm{TM}}$ Plus Direct cDNA Labeling System (Invitrogen) according to the manufacturer's instructions. Around $0.1 \mathrm{pmol}$ of $B$. subtillis gene specific primer mix was used to prime the cDNA synthesis. Labeled Alexa Fluor 555 and Alexa Fluor 647-cDNAs were purified with PCR purification kit (Qiagen). B. subtillis $168 \mathrm{cDNA}$ microarrays used in this study were obtained from Eurogentec. 

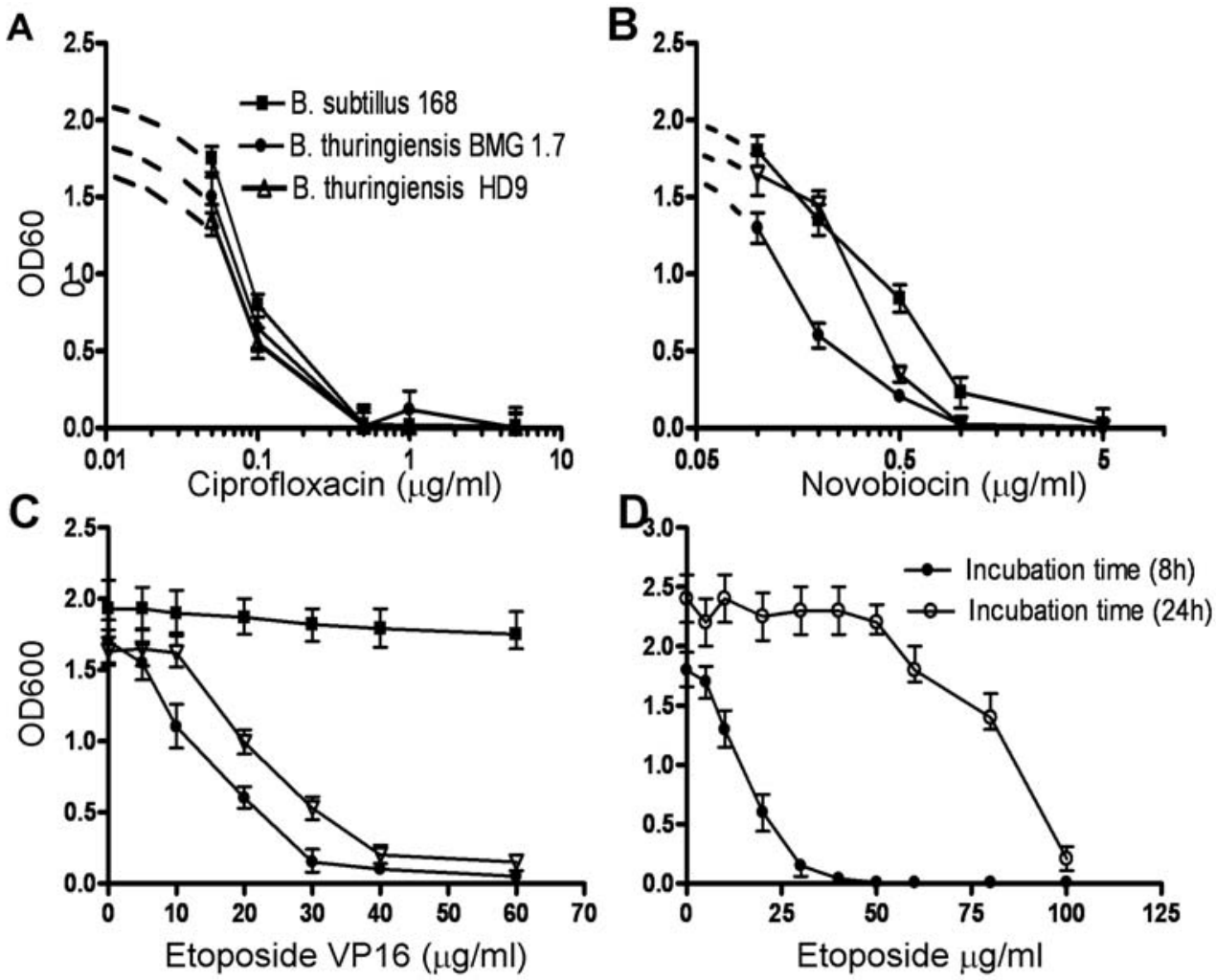

Figure 1. Effects of ciprofloxacin (A) and novobiocin (B) or etoposide (C) on Bacillus strains. Media containing various drug concentrations were inoculated with bacteria at an optical density of 0.4 . The optical density was measured after $7 \mathrm{~h}$ of incubation with the drugs at $37^{\circ} \mathrm{C}$. (D) Effect of VP16 on $B$. thuringiensis BGM 1.7 growth. Growth was measured at 8 and $24 \mathrm{~h}$ incubation time with VP16.

The hybridization and washing conditions were performed according to the manufacturer's instructions. Data preprocessing and normalization were performed as described previously (14). A linear model fitting and empirical Bayes methods were used for assessing differential gene expression using Bioconductor in R (15). The used decide tests classify a series of related t-statistics as up, down or not significant, multiple testing across genes and contrasts (1) $5 \mathrm{~min}$; (2) $20 \mathrm{~min}$ were used. The restriction of minimum 2-fold change and $p<0.05$ was applied. The Venn diagrams were prepared to visualize the intersections of the significant gene sets within groups. Hierarchical clustering of genes for visualization of expression patterns was performed in MultiExperiment Viewer (MEV) (16). Gene ontology (GO) web-based database JProGO was used for GO interpretation of prokaryotic microarray data (17).

\section{Results and discussion}

Effects of gyrase inhibitors and anti-tumor drugs on the growth of B. subtillis and thuringiensis. Among antibacterials in clinical use, the quinolones constitute the only group that target bacterial DNA topoisomerases, particularly DNA gyrase (3). These agents have no effect on the eukaryotic type II DNA topoisomerase, which is sensitive to two semisynthetic epipodophyllotoxins, etoposide VP16 and teniposide VM26. Coumarins such as novobiocin and coumermycin are also specific inhibitors of eubacterial DNA gyrase, but at low concentrations $(<10 \mu \mathrm{g} / \mathrm{ml})$. Higher concentrations also inhibit eukaryotic type II DNA topoisomerase.
In previous studies we have shown that halophilic archeabacteria are sensitive to antitumoral drugs active on the eukaryotic enzyme and to low doses of coumarins and fluoroquinolones specific for DNA gyrase (10). These findings, prompted us to investigate the sensitivity of three Bacillus strains to eubacterial and eukaryotic DNA topoisomerase inhibitors. First, we investigated the effects of novobiocin, ciprofloxacin, and etoposide VP16 on B. subtillis 168, Bacillus thuringiensis BMG 1.7, and HD9 strains. As shown in Fig. 1A, B and C, novobiocin and ciprofloxacin inhibited the growth of Bacillus subtillis 168 and Bacillus thuringiensis strains at low doses ( $\mathrm{IC}_{50}$ between 0.1 and $1 \mu \mathrm{g}$ ) $\mathrm{ml}$ ). In contrast to Bacillus subtillis 168, Bacillus thuringiensis strains are also sensitive to etoposide VP16 $\left(\mathrm{IC}_{50}, \sim 20 \mu \mathrm{g} / \mathrm{ml}\right)$, a specific inhibitor of eukaryotic type II DNA topoisomerase. Out of the 16 tested strains, B. thuringiensis toumanoffi (serotype 11a11b) was the most sensitive to VP16. We suspect that the varied behavior between the different $B$. species was probably due to their topoisomerase activity and/or permeability to the tested drugs.

Although the growth of $B$. thuringiensis was inhibited at low doses of VP16 during the 8-12 h incubation time, the growth rates of the tested strains were significantly restored after one night incubation ( $24 \mathrm{~h}$ ) with VP16 at concentrations $<80 \mu \mathrm{g} / \mathrm{ml}$. DNA plasmids isolated from the overnight cultures exhibited the same profiles as those isolated from the $8 \mathrm{~h}$ cultures, indicating that growth restoration was not due to bacterial contamination. Higher concentrations $(>80 \mu \mathrm{g} / \mathrm{ml})$ inhibited bacterial growth. Fig. 1D illustrates this phenomenon of drug tolerance induced in B. thuringiensis BGM 1.7 strain. 
A

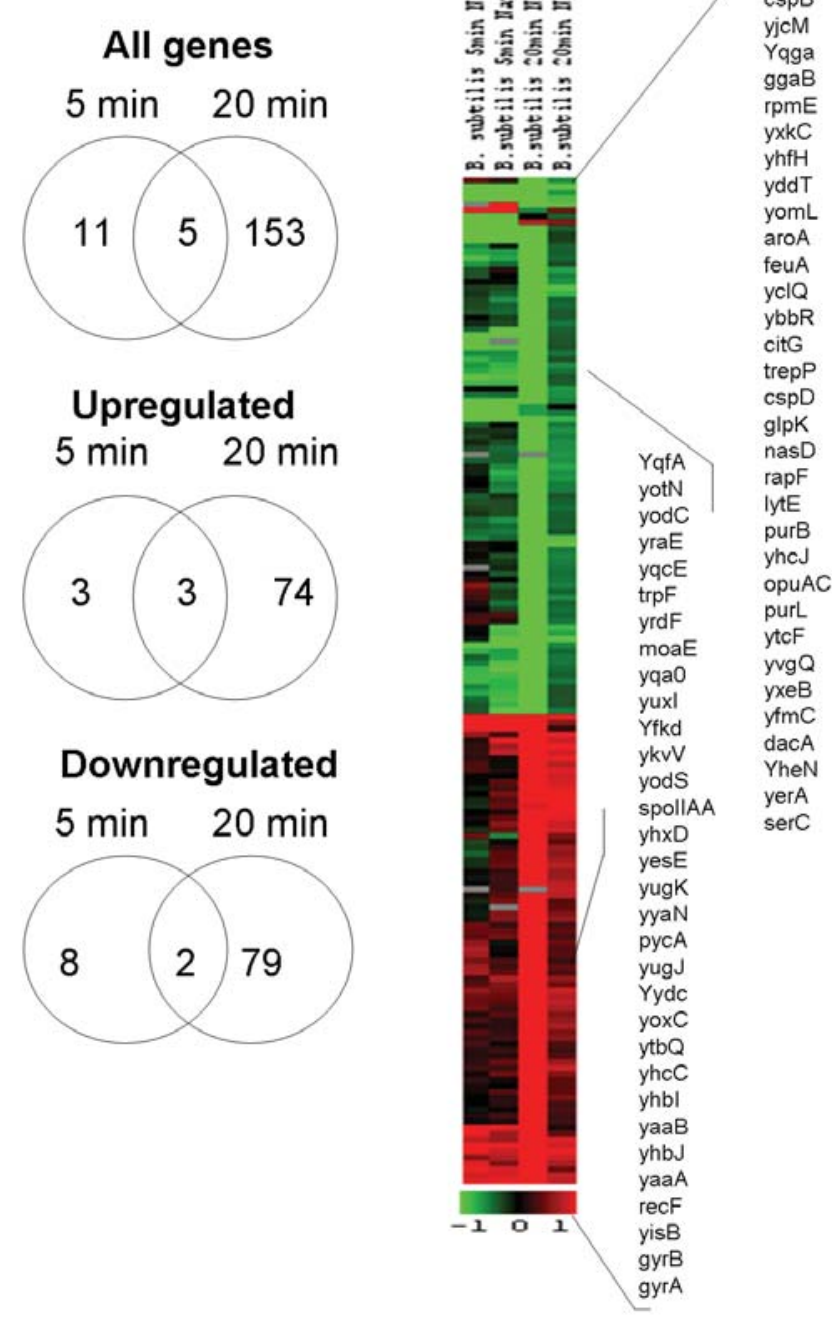

Figure 2. Analysis of novobiocin-induced gene expression in B. subtillis 168. (A) A Venn diagram of significantly altered genes at 5 and 20 min after novobiocin addition. Each circle represents one time-point as indicated. (B) Hierarchical clusters of genes that were significantly changed at $5 \mathrm{~min}$ and $20 \mathrm{~min}$ after novobiocin addition $(\mathrm{p}<0.05)$. Horizontal stripes represent genes and columns show treatment protocols. The log2-fold changes of gene ration are colour-coded as shown in the bar.

Re-growth occurred with thuringiensis toumanoffi at concentrations below $60 \mu \mathrm{g} / \mathrm{ml}$. Collectively, the data suggested the presence of active mechanisms by which DNA alterations in $B$. thuringiensis can be repaired and/or drug inactivated.

DNA relaxation affects gene expression. DNA supercoiling is a major regulator of transcription in bacteria. In eukaryotes stable changes in nucleosome structure have been correlated with transcriptionally active chromatin, whereas increases in DNA twist correlated with chromatin folding and transcriptional silencing (18). To maintain a physiological DNA supercoiling state, each cell has to regulate the expression of type I and type II DNA topoisomerases. The balanced activity of these enzymes results in a steady-state level of negative supercoiling that facilitates gene expression. In turn, supercoiling modulates the expression of the genes encoding for the DNA topoisomerases. We have previously shown that

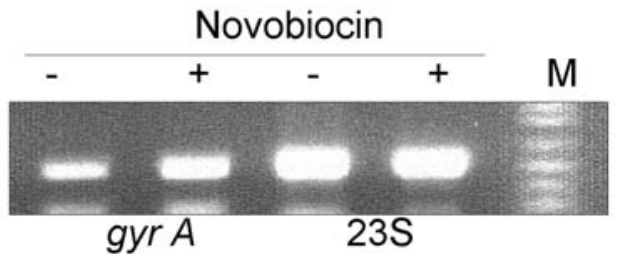

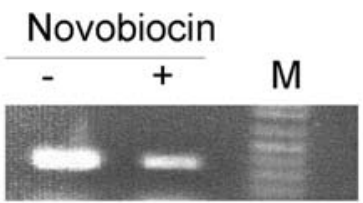

top $A$

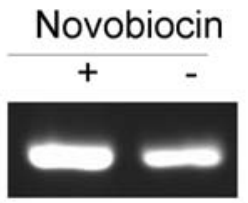

gyrB
Figure 3. RT-PCR analysis of gyr $A$, gyrB and top $A$ gene expression in untreated and novobiocin-treated $B$. subtillis 168. Cultures of B. subtillis at an optical density of 0.4 were supplemented with novobiocin $(10 \mu \mathrm{g} / \mathrm{ml})$. After 20 min drug-treatment, bacterial cells were harvested, total RNA was prepared, reverse transcribed, and then used as a template for PCR as described in materials and methods. M, $1 \mathrm{~kb}$ plus DNA ladder.

the inhibition of archaebacterial DNA topoisomerase II by either VP16 or novobiocin induced relaxation and positive supercoiling in plasmid DNA (11). In contrast to negative supercoiling, positive supercoiling is unfavourable to replication, transcription and recombination by increasing the stability of the DNA double helix. However, highly negatively supercoiled DNA would hinder several processes such as DNA strand separation required for replication and transcription. These observations underlie the biological significance of the simultaneous presence of two opposite DNA topoisomerase activities in each living cell.

To identify genes that are affected by DNA supercoiling, we used whole-genome microarrays to monitor the transcriptional response in $B$. subtillis 168 strain in response to novobiocin. In these experiments, exponentially growing cells were treated with novobiocin $(20 \mu \mathrm{g} / \mathrm{ml})$ for $5 \mathrm{~min}$ and 20 min. DNA I-treated total RNA isolated from untreated and treated cells was used to prepare fluorescently labeled cDNA transcripts which were hybridized to the arrays. To identify genes with significantly changed expression in treated cells compared to untreated cells, we used a linear model fitting and empirical Bayes methods (15). The restriction of minimum 2-fold change and $\mathrm{P}<0.05$ was also applied. Using this threshold, relatively few changes in gene expression were identified at early response $(5 \mathrm{~min})$, while the number of altered genes increased to 169 genes at 20 min after drug addition (Fig. 2A). Out of the 169 genes, 80 were increased and 89 were decreased in expression. A cluster of all significantly altered genes is presented in Fig. 2B. Overall, the inhibition of GyrB ATPase activity by novobiocin resulted in the induction SOS response genes, up-regulation of the gyrA and gyrB genes. Also, several $\mathrm{ABC}$ transporter genes were affected by novobiocin treatment. Genes encoding proteins involved in purine, glycine, serine, threonine, fructose, and mannose metabolisms were generally repressed. In contrast, gene encoding DNA replication and repair were significantly up-regulated (6-fold). Based on the expression data, the observed response to novobiocin can be rationalized as a 
Table I. Enriched GO pathways after novobiocin treatment.

\begin{tabular}{|c|c|c|c|}
\hline Category & GO Accession & GO Name & P-value \\
\hline MF & GO:0003918 & DNA topoisomerase (ATP-hydrolyzing) activity & $2.5099 \mathrm{E}-6$ \\
\hline MF & GO:0003916 & DNA topoisomerase activity & 8.7803E-6 \\
\hline BP & GO:0006265 & DNA topological change & $2.2979 \mathrm{E}-5$ \\
\hline $\mathrm{MF}$ & GO:0016853 & isomerase activity & $6.8492 \mathrm{E}-4$ \\
\hline $\mathrm{BP}$ & GO:0006259 & DNA metabolism & 0.0029 \\
\hline $\mathrm{BP}$ & GO:0006268 & DNA unwinding during replication & 0.0039 \\
\hline $\mathrm{BP}$ & GO:0006304 & DNA modification & 0.0094 \\
\hline $\mathrm{CC}$ & GO:0005694 & chromosome & 0.0118 \\
\hline $\mathrm{BP}$ & GO:0043283 & biopolymer metabolism & 0.0138 \\
\hline $\mathrm{BP}$ & GO:0006261 & DNA-dependent DNA replication & 0.0149 \\
\hline MF & GO:0005524 & ATP binding & 0.0157 \\
\hline $\mathrm{MF}$ & GO:0030554 & adenyl nucleotide binding & 0.0168 \\
\hline MF & GO:0017076 & purine nucleotide binding & 0.0194 \\
\hline $\mathrm{MF}$ & GO:0000166 & nucleotide binding & 0.0209 \\
\hline $\mathrm{MF}$ & GO:0003677 & DNA binding & 0.0238 \\
\hline $\mathrm{BP}$ & GO:0006260 & DNA replication & 0.036 \\
\hline MF & GO:0003676 & nucleic acid binding & 0.0428 \\
\hline
\end{tabular}

MF, molecular function; BP, biological process; CC, cellular compartment.

bacterial defence strategy to reduce intake of the drug, repair DNA damage, and restore DNA structure in the cell.

In order to explore the biological significance of novobiocin-mediated gene expression changes, we analyzed the Gene Ontology Annotations (17). This analysis highlighted the alteration of several functional pathways such as DNA topoisomerase activity, DNA replication, DNA topological change, and DNA metabolism (Table I). Collectively, the expression data indicate that supercoiling can function as a natural process with positive and negative effects on specific genes. It should be noted that when fold change of more than 1.5 was taken as the threshold for gene selection, around 1075 genes were affected by novobiocin at $20 \mathrm{~min}$, indicating that the expression of a large number of genes were affected by supercoiling at the lower fold change.

Alterations in gene expression in response to novobiocin were also evaluated by RT-PCR. These experiments were performed on samples treated for $20 \mathrm{~min}$ with the drugs. Fig. 3 shows a representative example of $g y r A$, gyr $B$, and top $A$ gene expression in novobiocin-treated $B$. subtillis 168 cells. The expression of gyrA and $g y r B$ genes was significantly increased after drug treatment, while top $A$ gene was decreased, confirming the microarray data. The ribosomal RNA 23S was used as an internal control since its expression was not significantly affected by novobiocin during the $20 \mathrm{~min}$ incubation time. Collectively, the data confirm previous studies investigating the effects of novobiocin on DNA gyrase activity $(19,20)$.

Effects of novobiocin and VP16 on gene expression in $B$. turingiensis. The sensitivity of $B$. turingiensis to eubacterial DNA gyrase and eukaryotic type II DNA topoisomerase inhibitors prompted us to investigate the effects of these agents on gene expression. We looked at the effects of novobiocin, ciprofloxacin, and VP16 on the expression of the gyrA gene by RT-PCR. Fig. 4A shows a representative example of gyrA, gene expression in novobiocin-, ciprofloxacin-, and VP16-treated cells. A significant increase in gyrA mRNA levels was evident in novobiocin- or ciprofloxacin- treated cells. VP16 also affected the expression of the gyrA gene, although the effect was modest. Based on 4 experiments, the deregulation levels observed in novobiocin- or ciprofloxacin-treated cells were between 2 and 10-fold versus untreated cells, whereas those seen in VP16 treated cells were $\sim 0.5$-fold change. Furthermore, the gene encoding for the main enzyme involved in DNA relaxation, top $A$ was down-regulated in novobiocin-treated cells but not in VP16 treated cells (Fig. 4B). Collectively, the data indicate that at the used concentration $(50 \mu \mathrm{g} / \mathrm{ml}) \mathrm{VP} 16$ has a modest transcriptional effect on $g y r A, g y r B$, and top $A$ gene expression despite the inhibition of cell growth.

Activation of the SOS system by VP16 and novobiocin. In the absence of DNA damage, the SOS genes are each directly repressed by the binding of the LexA protein to SOS operator sites (21). Induction of the SOS genes following, for example, DNA damage, generally occurs when RecA protein activated by a signal generated through the processing of damaged DNA, promotes the proteolytic destruction of LexA protein (21). If VP16 induces growth arrest and possibly DNA damage as illustrated in Fig. 1, then we would predict the induction of the SOS response to DNA damage. Consequently, we have analyzed the expression of $\operatorname{din} B$ and $\operatorname{lex} A$ genes in the VP16-treated $B$. thuringiensis BGM 1.7 strain. As shown in Fig. 5, VP16 treatment resulted in the induction of $\operatorname{din} B$ gene and suppression of lexA gene at $60 \mathrm{~min}$ incubation time, 
A

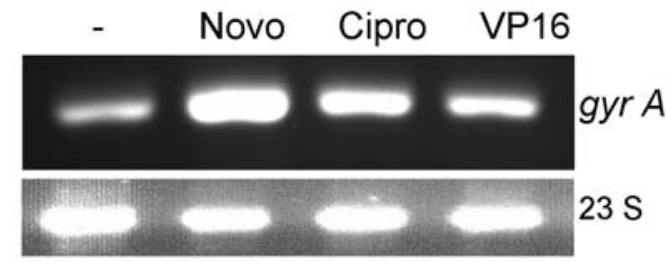

B

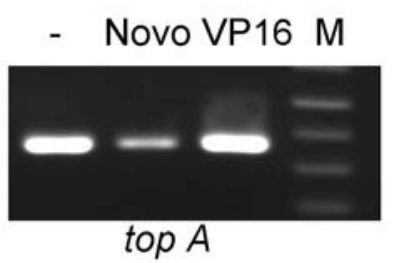

Figure 4. RT-PCR analysis of gyrA and topA gene expression in B. turingiensis $B G M 1.7$ in response to novobiocin, ciprofloxacin or VP16. Culture conditions and drug treatment are as in Fig. 3.

thus supporting the induction of DNA damage. In B. subtillis 168-treated novobiocin, the microarray data indicate that the expression of the lexA gene was significantly down-regulated (5-fold reduction) compared to untreated cells. The expression of the recA gene was not changed by novobiocin, however, its counterpart $r e c F$ was significantly induced (5.5 fold increase). $\mathrm{RecF}$ and $\mathrm{RecO}$ proteins are involved in DNA recombination and play an important role in DNA double-strand break repair in B. subtillis (22). Collectively, the microarray expression data indicate that novobiocin also activates the SOS system in B. subtillis.

Effects of novobiocin and VP16 on plasmid topology in the $B$. thuringiensis 1.7 strain. In eubacteria, the level of DNA supercoiling is controlled by the antagonistic activities of DNA gyrase and protein $\omega$. Inhibition of DNA gyrase in vivo alters the balance between the two DNA topoisomerase activities, thus reducing the super helical density of chromosomal and plasmid DNA. Usually plasmids are used to detect drug effects on DNA topology. In this respect, we previously demonstrated that plasmid isolated from etoposide or novobiocin-treated Halobacterium GRP was negatively and positively supercoiled $(11,23)$. In the course of this study, we noted the presence of a small plasmid $(\sim 2 \mathrm{~kb})$ in the $B$. thuringiensis 1.7 strain. Therefore, this plasmid was used as a marker in order to monitor changes in DNA supercoiling. Fig. 6 compares the electrophoretic mobilities of plasmid DNA isolated from cells treated or not with either novobiocin or etoposide VP16. As shown, the $2 \mathrm{~kb}$ plasmid isolated from cells treated with novobiocin for $1 \mathrm{~h}$ is relaxed and composed of gradually relaxed topoisomers, indicating that in $B$. thuringiensis novobiocin inhibits DNA gyrase which maintains the $2 \mathrm{~kb}$ plasmid in a negatively supercoiled state (form I), and this inhibition reveals another activity of DNA topoisomerase I, which increases the linking number of this plasmid. In contrast to novobiocin, no significant activity of VP16 on plasmid topology was detected, although more sensitive methods such as autoradiography might sense some topological changes. It should be noted that topological changes in high molecular-weight plasmid DNA cannot be easily monitored in $1 \%$ agarose gels. However, as indicated by

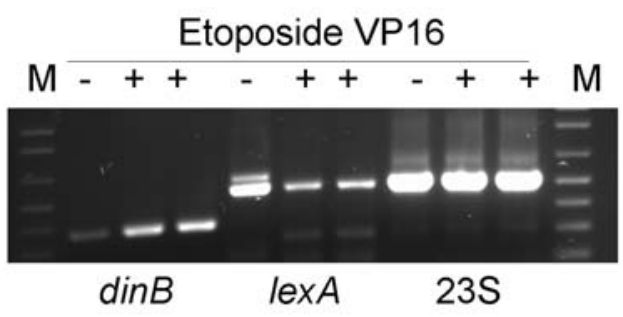

Figure 5. RT-PCR analysis of $\operatorname{din} B$ and lexA gene expression in B. turingiensis BGM 1.7 in response to VP16. Culture conditions and drug treatment are as in Fig. 3. M, 1 kb plus DNA ladder.

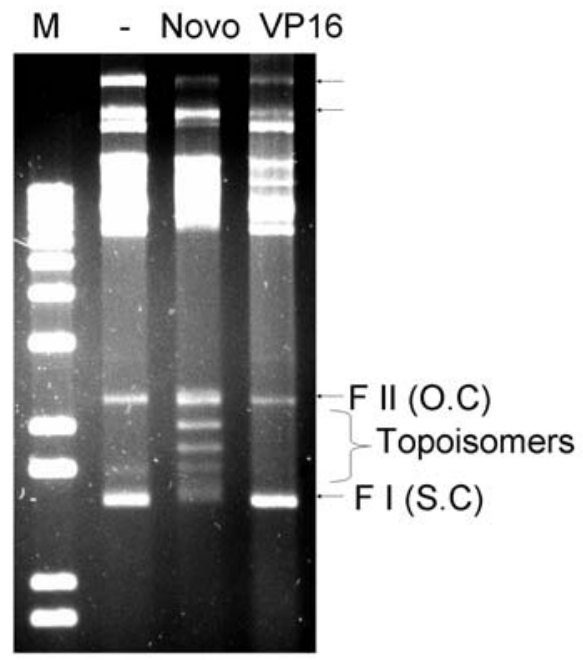

Figure 6. Analysis of plasmid supercoiling in B. turingiensis. Cultures of B. turingiensis BGM 1.7 at an optical density of 0.4 were supplemented with either novobiocin $(10 \mu \mathrm{g} / \mathrm{ml})$ or etoposide VP16 $(50 \mu \mathrm{g} / \mathrm{ml})$ and incubated at $37^{\circ} \mathrm{C}$ for $1 \mathrm{~h}$. Subsequently, plasmid DNA was prepared from treated and untreated cells and then analysed using $1 \%$ agarose gel. After electrophoresis the gel was stained with ethidium bromide. OC, open circular; SC, supercoil circular; M, $1 \mathrm{~kb}$ plus DNA ladder.

the upper arrows some high molecular plasmid and/or phage DNAs were affected by novobiocin or VP16 treatment. Of note, polyethylene glycol precipitation of possible secreted phages suggests that phage replication and packaging are inhibited in treated cells.

In summary, the present study provides valuable insights into the processes altered in B. subtillis and B. thuringiensis in response to DNA topoisomerase inhibitors and shows the usefulness of the $2 \mathrm{~kb}$ plasmid in monitoring topological changes in vivo. The study also underscores the induction of the SOS system by VP16 in B. thuringiensis BMG 1.7 strain. It will be useful to provide more insights into the mechanisms of VP16-induced drug tolerance. Drug-tolerance was also observed with novobiocin and ciprofloxacin at low concentrations.

\section{Acknowledgements}

We thank Dr Anne Dybwad for critical reading of the manuscript and Abir Belaouis for bringing the bacterial strains. This research was supported by the Norwegian Cancer Society to M. Sioud. 


\section{References}

1. Champoux JJ: DNA topoisomerases: structure, function, and mechanism. Annu Rev Biochem 70: 369-413, 2001.

2. Forterre P, Gribaldo S, Gadelle D and Serre M-C: Origin and evolution of DNA topoisomerases. Biochimie 89: 427-446, 2007.

3. Hooper DC: Mechanisms of action of antimicrobials: focus on fluoroquiolones. Antimicrob Activity 32: S9-S15, 2001.

4. Burden DA and Osheroff N: Mechanism of action of eukaryotic topoisomerases and topoisomerase II and drug targeted to the enzyme. Biochem Biophys Acta 1400: 139-154, 1998.

5. Chen GL, Yang L, Rowe TC, Halligan BD, Tewey KM and Liu LF: Nonintercalative antitumor drugs interfere with the breakage-reunion reaction of mammalian DNA topoisomerase II. J Biol Chem 259: 13560-13566, 1984.

6. Mosesso P, Darroudi F, Van Den Berg M, Vermeulen S, Palitti F and Natarajan AT: Induction of chromosomal aberrations (unstable and stable) by inhibitors of topoisomerase II, mAMSA and VP16, using conventional Giemsa staining and chromosome painting techniques. Mutagenesis 13: 39-43, 1998.

7. Sioud M, Possot O, Elie C, Sibold L and Forterre P: Coumarin and quinolone action in archaebacteria: Evidence for the presence of a DNA gyrase-like enzyme. J Bacteriol 170: 946-953, 1988.

8. Sioud M, Baldacci G, Forterre P and de Recondo AM: Antitumor drug inhibit the growth of halophilic archaebacteria. Eur J Biochem 169: 231-236, 1987.

9. Woese CR: Bacterial evolution. Microbiol Rev 51: 221-227, 1987.

10. Sioud M and Forterre P: Ciprofoxacin and etoposide (VP16) produce a similar pattern of DNA cleavage in a plasmid of an Archaebacterium. Biochem 28: 3638-3641, 1989.

11. Sioud M, Forterre P and de Recondo AM: Effects of the antitumor drug VP16 (etoposide) on the archaebacterial Halobacterium GRB $1.7 \mathrm{~kb}$ plasmid in vivo. Nucleic Acids Res 15: 8217-8234, 1987.

12. Cherif A, Ouzari H, Daffonchio D, Cherif H, Ben Slama K, Hassen A, Jaoua S and Boudabous A: Thuricin 7: a novel bacteriocin produced by Bacillus thuringiensis BMG1.7, a new strain isolated from soil. Lett Appl Microbiol 32: 243-247, 2001.

13. Cherif A, Chehimi S, Limem F, Hansen BM, Hendriksen NB, Daffonchio D and Boudabous A: Detection and characterization of the novel bacteriocin entomocin 9, and safety evaluation of its producer, Bacillus thuringiensis ssp. Entomocidus HD9. J Appl Microbiol 95: 990-1000, 2003.
14. Cekaite L, Furset G, Hovig E and Sioud M: Gene expression analysis in blood cells in response to unmodified and 2'modified siRNAs reveals TLR-dependent and independent effects. J Mol Biol 365: 90-108, 2007.

15. The R Project for Statistical Computing (http://www.rproject.org).

16. TM4 microarray software suite (http://www.tm4.org/).

17. Scheer M, Klawonn F, Munch R, Grote A, Hiller K, Choi C, Koch I, Schobert M, Hartig E, Klages U and Jahn D: JProGO: a novel tool for the functional interpretation of prokaryotic microarray data using Gene Ontology information. Nucleic Acids Res 34(Web Server issue):W510-W515, 2006.

18. Travers A and Muskhelishvili G: A common topology for bacterial and eukaryotic transcription initiation? EMBO Rep 8: 147-151, 2007.

19. Jeong KS, Xie Y, Hiasa H and Khodursky AB: Analysis of pleiotropic transcriptional profiles: a case study of DNA gyrase inhibition. PLoS Genet 2: 1464-1476, 2006.

20. Gmuender H, Kuratli K, Di Padova K, Gray CP, Keck W and Evers S: Gene expression changes triggered by exposure of Haemophilus influenzae to novobiocin or ciprofloxacin: combined transcription and translation analysis. Genome Res 11: $28-42,2001$

21. Au N, Kuester-Schoeck E, Mandava V, Bothwell LE, Canny SP, Chachu K, Colavito SA, Fuller SN, Groban ES, Hensley LA, O'Brien TC, Shah A, Tierney JT, Tomm LL, O'Gara TM, Goranov AI, Grossman AD and Lovett CM: Genetic composition of the Bacillus subtilis SOS system. J Bacteriol 187: 7655-7666, 2005.

22. Kidane D, Sanchez H, Alonso JC and Graumann PL: Visualization of DNA double-strand break repair in live bacteria reveals dynamic recruitment of Bacillus subtilis RecF, RecO and RecN proteins to distinct sites on the nucleoids: Mol Microbiol 52: 1627-1639, 2004.

23. Sioud M, Baldacci G, de Recondo AM and Forterre P: Novobiocin induces positive supercoiling of small plasmids from halophilic archaebacteria in vivo. Nucleic Acids Res 16: 1379-1391, 1988. 